\title{
Sustainability Assessment of Regional Water Resources Use Based on PSO-PPE
}

\author{
Qiang Fu, Qiuxiang Jiang, and Zilong Wang \\ College of Water Conservancy \& Architecture, Northeast Agricultural University, \\ Harbin, P.R. China 150030 \\ fuqiang@neau.edu.cn, \{jiangqiuxiang914,wz11216\}@163.com
}

\begin{abstract}
Aiming at poor valuing objectivity for indicator weight in sustainability assessment of water resources use (SAWRU), an indicator system and a projection pursuit evaluation model optimized by particle swarm optimization algorithm were structured for SAWRU, and then the method was applied to the SAWRU of Sanjiang Plain. The applied results indicated that the method not only avoided artificial disturbance when valuing indicator weight but also had the advantages of objective and precise assessment result, feasibility and availability. The assessment results showed that the sustainable grade of water resources use of Jiamusi, Shuangyashan and Jixi which located at Sanjiang Plain belonged to III, and the grade of the other regions of Sanjiang Plain belonged to IV. Thus, scientific water resource use scheme and reasonable socioeconomic development strategy need to be made based on the regional characteristics of water resources and socioeconomic development.
\end{abstract}

Keywords: Water resources sustainable utilization, Projection pursuit evaluation, Particle swarm optimization, Sanjiang Plain.

\section{Introduction}

Sustainability assessment of water resources use (SAWRU) is the key problem of water resources sustainability utilization research, the essential means for measuring water resources sustainable capacity and the main foundation of macro adjustment for regional water resources [1]. Presently, scholars, at home or abroad, usually use comprehensive grading method, fuzzy comprehensive evaluation method, grey clustering evaluation method, principal component analysis and factor analysis method to evaluate water resources sustainable utilization. However, human factors exist in the assignment of evaluation indicator weight of the above methods and cause poor objectivity of assessment results. For SAWRU with multiple indicators, the influence degree of each evaluation indicator on water resources sustainable utilization should be correctly analyzed under the least human factor effect, and then a comprehensive evaluation result reflecting the characteristic information of each indicator can be obtained. Projection pursuit evaluation (PPE) model is an exploratory data analysis method which drove by sample data [2]. The method firstly pursues the best projection direction on the basis of sample data characteristics, next judges the 
contribution degree of each evaluation indicator to comprehensive evaluation target based on the directions, and then obtains the projection values according to the best projection direction and the linear projection of evaluation indicators, comprehensively assesses water resources sustainable utilization with the projection values at last [3]. Thus, the paper used projection pursuit evaluation model based on particle swarm optimization (PSO-PPE) to assess regional water resources sustainable utilization and provided a new and effective evaluation method to the research of water resources utilization.

\section{Methods}

\subsection{Projection Pursuit Evaluation Model}

The modeling steps of projection pursuit evaluation (PPE) model based on assessment criterion of water resources sustainable utilization are as follows [4] and [5]:

Step 1: Normalizing the evaluation indicators of each sample

Set the classification criterion sample set of the SAWRU indicators as $\left\{x^{*}(i, j) \mid i=1 \sim n, j=1 \sim p\right\}$, where $x^{*}(i, j)$ is the indicator $j$ value of sample $i, n$ the number of samples and $p$ the number of indicators. In order to eliminate the dimension influence and unify the variable scope of each indicator value, we can adopt the following formulas to normalize the extreme value:

$$
x(i, j)= \begin{cases}\frac{x^{*}(i, j)}{x_{\max }(j)} & \text { in case the bigger the indicator the better } \\ \frac{x_{\min }(j)}{x^{*}(i, j)} & \text { in case the smaller the indicator the better }\end{cases}
$$

where $x_{\max }(j)$ and $x_{\min }(j)$ stand for the maximum and the minimum value of indicator $j$, respectively, and $x(i, j)$ stands for the array of normalized indicator characteristic value in the classification criterion sample set.

Step 2: Constructing the projection indicator function $Q(a)$

Projection pursuit model is to integrate $p$-dimensional data, $\{x(i, j) \mid j=1 \sim p\}$, into one-dimension projection value $z(i)$ :

$$
z(i)=\sum_{j=1}^{p} a(j) x(i, j) \quad(i=1,2, \cdots, n)
$$

based on projection direction $a=\{a(1), a(2), a(3), \cdots, a(p)\}$, where $a$ stands for unit length vector.

We then make a classification in accordance with one-dimension dispersion pattern of $\{z(i) \mid i=1 \sim n\}$. A good classification for the method requires the local projection dots as close as possible and the integral projection dot groups as dispersive. Namely, the standard deviation and partial density values of multiple data interspersing among 
one-dimension space should be maximized at the same time. Thus, the projection indicator function can be expressed as follows:

$$
Q(a)=S_{z} D_{z}
$$

where $S_{z}$ is the standard deviation of $z(i)$ and $D_{z}$ the partial density of $z(i)$. Then we get

$$
\begin{gathered}
S_{z}=\sqrt{\frac{\sum_{i=1}^{n}(z(i)-E(z))^{2}}{n-1}} \\
D_{z}=\sum_{i=1}^{n} \sum_{j=1}^{n}(R-r(i, j)) \cdot u(R-r(i, j))
\end{gathered}
$$

where $E(z)$ is the average value of series $\{z(i) \mid i=1 \sim n\}, R$ is the window radius of partial density, which can be settled by test, but is assumed to equal $0.1 S_{z}$ in normal practice, $r(i, j)$ is the distance between the samples, $r(i, j)=|z(i)-z(j)|$, and $u(R-r(i, j))$ is a unit step function. Let $t=R-r(i, j)$, then $u(t)=1$ if $t \geq 0$ and $u(t)=0$ if $t<0$.

Step 3: Optimizing the projection indicator function

We can calculate the maximum value of $Q(a)$ to estimate the best project direction with restricted condition

$$
\sum_{j=1}^{p} a^{2}(j)=1
$$

It is a complex non-linearity optimization, in which $\{a(j) \mid j=1 \sim p\}$ is taken as the optimized variable. Therefore, it is quite difficult to make calculations with the traditional method. We now may adopt particle swarm optimization (PSO) algorithm.

Step 4: Grade assessment of water resources sustainable utilization

We put the best projection direction $a^{*}$ into Equation 2, and then we obtain the best projection value $z_{s}^{*}(i)$ of the separation dots of each grade in the classification criterion sample data. We normalize the indicator sample data of water resources sustainable utilization in the evaluated region and put the normalized data into Equation 2, and then we obtain the projection value $z^{*}(i)$ of the evaluated sample. Comparing $z^{*}(i)$ with $z_{s}^{*}(i)$, we obtain the grade that the regional water resources sustainable utilization belongs to.

\subsection{Particle Swarm Optimization Algorithm}

Particle swarm optimization (PSO) algorithm was proposed by Kennedy and Eberhart in 1995 [6] and [7], which is a kind of adaptive evolutionary computation technology 
and swarm intelligence algorithm. PSO algorithm considers each solution of optimization problem as a particle among the searching space and each particle have its own position, speed and adaptive value (fitness) decided by a certain optimization problem. The best position of each particle during the flight is the best solution found by the particle (the individual extremum, pbest), and then the best position experienced by the whole group is the best solution found by the whole group presently (the global extremum, gbest). Every particle constantly updates itself through pbest and gbest, and then a new population is created. At last, the whole population can comprehensively search the solution region [8].

Set the particle population size as $N$. The position of the $i$ th particle $(i=1,2, \cdots, N)$ can be expressed as $x_{i}$, speed as $v_{i}$ and adaptive value as $f_{i}$. During each iteration after the initial position and speed generate randomly, a particle updates itself by following the individual extremum $\operatorname{pbest}_{i}(t)$ and the global extremum gbest $(t)$. At the moment $t+1$, any particle $i$ can update its own position and speed by the following equations:

$$
\begin{gathered}
v_{i}(t+1)=w v_{i}(t)+c_{1} r_{1}(t)\left(\text { pbest }_{i}(t)-x_{i}(t)\right)+c_{2} r_{2}(t)\left(\text { gbest }(t)-x_{i}(t)\right) \\
x_{i}(t+1)=x_{i}(t)+v_{i}(t+1)
\end{gathered}
$$

where $c_{1}$ and $c_{2}$ are constants and called as learning factor, $r_{1}$ and $r_{2}$ are random numbers changing in the interval of $(0,1)$ and $w$ is inertia weight.

Then the individual extremum of each particle and global extremum of the whole particles can update by the equations as follows:

$$
\begin{aligned}
\operatorname{pbest}_{i}(t+1)= & \begin{cases}x_{i}(t+1) & f_{i}(t+1) \geq f\left(\text { pbest }_{i}(t)\right) \\
\text { pbest }_{i}(t) & f_{i}(t+1)<f\left(\text { pbest }_{i}(t)\right)\end{cases} \\
& \operatorname{gbest}(t+1)=x_{\max }(\mathrm{t}+1)
\end{aligned}
$$

where $f_{i}(t+1)$ is the adaptive value of $i$ particle at $t+1$ moment, $f\left(\right.$ pbest $\left._{i}(t)\right)$ is the best adaptive value of $i$ particle in its searching history, and $x_{\max }(t+1)$ is the position of the particle among all the particles corresponded with the biggest $f\left(\right.$ pbest $\left._{i}(t)\right)$ at $t+1$ moment. The adaptive value $f_{i}$ of each particle is decided by an actual optimization.

\section{Sustainable Assessment of Regional Water Resources Use}

\subsection{Study Area}

Sanjiang Plain is located in the northeast of Heilongjiang Province $\left(\mathrm{N} 45^{\circ} 01^{\prime} \sim 48^{\circ} 27^{\prime} 20^{\prime \prime}, \mathrm{E} 130^{\circ} 13^{\prime} \sim 135^{\circ} 5^{\prime} 19^{\prime \prime}\right)$ with the total area of $10.9 \times 10^{4} \mathrm{~km}^{2}$, which boundary is the Heilongjiang River to the north, the Xingkai lake to the south, 
the east side of Xiao Hinggan Mountains to the west and the Wusuli River to the east. The cities and towns located in the region include Jixi, Hegang, Shuangyashan, Jiamusi, Qitaihe, Muling and Yilan. Sanjiang Plain has flat terrain, fertile soil, well climate and abundant water resources which natural conditions are suitable for agricultural development. After years of exploitation and construction, Sanjiang Plain has become the important commodity grain production base of China. Thus, a scientific assessment of sustainable utilization degree for water resources in Sanjiang Plain has the important realistic significance.

\subsection{Establishing Indicator System for SAWRU}

According to the comprehensive analysis of water resources influencing factors in Sanjiang Plain and the indicator system of supply and demand analysis of water resources in China [9], the indicator system for SAWRU of the region was established and shown in Table 1. The indicator system includes seven indicators (irrigation ration $x_{1}$, water resources use ration $x_{2}$, water resources development degree $x_{3}$, water supply modulus $x_{4}$, water demand modulus $x_{5}$, per capita water supply quantity $x_{6}$, environment water use ratio $x_{7}$ ). For the alternate use of average and interval of the classification criterion for SAWRU in reference [10] made the classification partition undefined, we gave five normalized values in the form of interval for the seven indicators of SAWRU in the study and the criterion was shown in Table 1.

Table 1. Classification criterion for sustainable assessment of water resources use

\begin{tabular}{cccccccc}
\hline No. & Indicator & Type & Grade I & Grade II & Grade III & Grade IV & Grade V \\
\hline 1 & $x_{1}(\%)$ & positive & $>60$ & $45 \sim 60$ & $35 \sim 45$ & $20 \sim 35$ & $<20$ \\
2 & $x_{2}(\%)$ & positive & $>60$ & $45 \sim 60$ & $35 \sim 45$ & $20 \sim 35$ & $<20$ \\
3 & $x_{3}(\%)$ & positive & $>70$ & $55 \sim 70$ & $45 \sim 55$ & $30 \sim 45$ & $<30$ \\
4 & $x_{4}\left(10^{4} \mathrm{~m}^{3} / \mathrm{km}^{2}\right)$ & positive & $>100$ & $80 \sim 100$ & $60 \sim 80$ & $40 \sim 60$ & $<40$ \\
5 & $x_{5}\left(10^{4} \mathrm{~m}^{3} / \mathrm{km}^{2}\right)$ & positive & $>100$ & $80 \sim 100$ & $60 \sim 80$ & $40 \sim 60$ & $<40$ \\
6 & $x_{6}\left(\mathrm{~m}^{3}\right.$ per capita) & opposite & $<1000$ & $1000 \sim 1750$ & $1750 \sim 2250$ & $2250 \sim 3000$ & $>3000$ \\
7 & $x_{7}(\%)$ & opposite & $<2$ & $2 \sim 3$ & $3 \sim 4$ & $4 \sim 5$ & $>5$ \\
\hline
\end{tabular}

\subsection{Evaluation Results and Analysis}

The projection pursuit evaluation model based on particle swarm optimization algorithm (PSO-PPE) was programmed by MATLAB 7.0 software. In the program, we set the population size of PSO $N$ as 400, the learning factors $c_{1}$ and $c_{2}$ as 2 , the inertia weight $w$ as 0.9965 and the maximum iteration time $G_{\max }$ as 50 . Next we put the criterion and regional statistical data of SAWRU shown in Table 2 into the model 
and ran the PSO-PPE program under MATLAB environment. After that we obtained the best projection directions $a^{*}=(0.398,0.398,0.341,0.366,0.366,0.404,0.369)$, and then we put $a^{*}$ into Equation 2 to calculate the best projection values of the classification criterion date set $z_{s}^{*}=(2.642,1.927,1.487,0.986)$. Name, the sample for evaluation belongs to Grade I if $z^{*}>2.642$, belongs to Grade II if $1.927 \leq z^{*} \leq 2.642$, belongs to Grade III if $1.487 \leq z^{*} \leq 1.927$, belongs to Grade IV if $0.986 \leq z^{*} \leq 1.487$ and belongs to Grade V if $z^{*}<0.986$.

Considering Jixi, Hegang, Shuangyashan, Jiamusi, Qitaihe, Muling and Yilan located in Sanjiang Plain as the samples for evaluation of SAWRU and putting them into the above program, we obtained the best projection values $z^{*}$ of the seven regions for evaluation (shown in Table 2). Comparing it with the projection values of assessment criterion, we can judge which grade the water resources sustainable utilization of the regions for evaluation belong to.

Table 2. Indicator statistics value and the best projection value of SAWRU

\begin{tabular}{ccccccccc}
\hline Indicator & $x_{1}(\%)$ & $x_{2}(\%)$ & $x_{3}(\%)$ & $x_{4}\left(10^{4} \mathrm{~m}^{3} / \mathrm{km}^{2}\right)$ & $x_{5}\left(10^{4} \mathrm{~m}^{3} / \mathrm{km}^{2}\right)$ & $\begin{array}{c}x_{6}\left(\mathrm{~m}^{3} \text { per }\right. \\
\text { capita })\end{array}$ & $x_{7}(\%)$ & $z^{*}$ \\
\hline Jixi & 16.16 & 39 & 70.60 & 14.11 & 15.67 & 1663 & 0.52 & 1.874 \\
Hegang & 12.91 & 33 & 32.88 & 8.07 & 12.52 & 1082 & 0.36 & 1.465 \\
Shuangyashan & 12.86 & 40 & 53.00 & 8.89 & 12.47 & 1302 & 0.24 & 1.741 \\
Jiamusi & 18.82 & 38 & 82.17 & 13.16 & 18.25 & 1710 & 0.89 & 1.927 \\
Qitaihe & 3.07 & 35 & 15.69 & 2.11 & 2.98 & 145 & 0.35 & 1.215 \\
Muling & 1.44 & 43 & 5.53 & 1.10 & 1.40 & 219 & 0.23 & 1.144 \\
Yilan & 6.09 & 37 & 28.95 & 6.71 & 5.90 & 766 & 0.49 & 1.133 \\
\hline
\end{tabular}

On the basis of the projection values of assessment criterion and the visual function of ArcGIS software, a spatial distribution map of evaluation grades for water resources sustainable utilization of Sanjiang Plain was drew and shown in Figure 1. In the region of Sanjiang Plain, the water resources sustainable utilization of Jiamusi, Shuangyashan and Jixi belonged to Grade III, which indicated that a balance existed between water resources sustainable utilization and socioeconomic development, but along with the rapid growth of economics and the increase of population size, water resources demand will increase day by day, thus making the best of the current water resources, strengthening management and exploiting water resources deeply were important to ensure a sustainable supply capacity of water resources for the socioeconomic development of these regions. However, the water resources sustainable utilization of Hegang, Yilan, Qitaihe and Muling belonged to Grade IV, which showed a low level of sustainable utilization, but the current exploitation degree and use ration of water resources of these regions were low, a good prospect for water resources sustainable utilization can be saw if increasing construction 
investment of water conservancy project and adopting strict water saving measures for industry and agriculture to enhance the water resources exploitation degree of these regions.

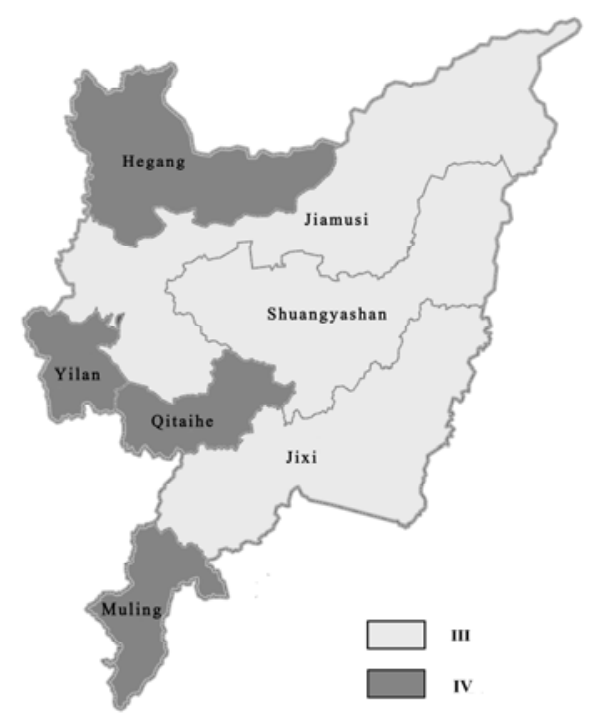

Fig. 1. Spatial distribution map of evaluation grades for regional water resources sustainable utilization

\section{Conclusions}

The PSO-PPE model for SAWRU cluster and evaluate the samples based on their own characteristics and do not need indicators' weight prior, which avoid human randomicity. At the same time, intuitiveness and good maneuverability are also the advantages of the model. The PSO-PPE model has been applied successfully in the SAWRU of Sanjiang Plain, and it was proved a feasible method and provided a new way for SAWRU with multiple influencing factors.

In the region of Sanjiang Plain, the SAWRU of Jiamusi, Shuangyashan and Jixi was Grade III showing a balance between water resources use and socioeconomic development. However, the SAWRU of Hegang, Yilan, Qitaihe and Muling was Grade IV showing a low level of water resources sustainable utilization capacity, thus a scientific exploitation and utilization plan for water resources combining the characteristics of the resources and socioeconomic development of these regions was required exigently.

Acknowledgements. The study was supported by the Program for New Century Excellent Talents In Heilongjiang Provincial University (1155-NCET-004). 


\section{References}

1. Song, S., Cai, H.: Bossel indicator system and assessment method for sustainable utilization of regional water resources. Journal of Hydraulic Engineering 6, 68-74 (2004)

2. Friedman, J.H., Turkey, J.W.: A projection pursuit algorithm for exploratory data analysis. IEEE Transactions On Computer 9, 881-890 (1974)

3. Feng, Z., Zheng, H., Liu, B.: Comprehensive evaluation of agricultural water use efficiency based on genetic projection pursuit model. Transactions of the CSAE 3, 66-70 (2005)

4. Zhao, X., Fu, Q., Xing, Z.: Improvement of projection pursuit model and the application in integrated evaluation of eco-agricultural construction. Transactions of the CSAE 5, 222-225 (2006)

5. Wang, S., Hou, Y., Zhang, X.: Comprehensive evaluation method for water resources carrying capacity in river basins. Journal of Hydraulic Engineering 1, 88-92 (2003)

6. Kennedy, J., Eberhart, R.C.: Particle Swarm Optimization. In: IEEE International Conference on Neural Networks, pp. 1942-1948 (1995)

7. Eberhart, R.C., Kennedy, J.: A New Optimization Using Particle Swarm Theory. In: Proceedings of the Sixth International Symposium on Micro Machine and Human Science, pp. 39-43 (1995)

8. Wang, Z., Fu, Q., Jiang, Q.: Soil nutrient management zones based on particle swarm optimization algorithm. Transactions of the CSAE 10, 80-84 (2008)

9. Planning and Design Institute of Water Conservancy and Hydropower, Ministry of Water Conservancy and Electric Power: Water Resources Utilization in China. Water Resources and Electric Power Press, Beijing, 114-134 (1989)

10. Chen, S.: Theory model and a method for qualitative assessment of sustainable development of regional water resources. Engineering Science 2, 33-38 (2001) 